\title{
Eye movement monitoring reveals differential influences of emotion on memory
}

\author{
Lily Riggs ${ }^{1,2 *}$, Douglas A. McQuiggan' , Adam K. Anderson ${ }^{1,2}$ and Jennifer D. Ryan ${ }^{1,2,3}$ \\ 1 Rotman Research Institute, Toronto, ON, Canada \\ 2 Department of Psychology, University of Toronto, Toronto, ON, Canada \\ ${ }^{3}$ Department of Psychiatry, University of Toronto, Toronto, ON, Canada
}

\section{Edited by:}

Jorge L. Armony, McGill University,

Canada

\section{Reviewed by:}

Kevin S. Labar, Duke University, USA Andrew Yonelinas, University of

California Davis, USA

\section{${ }^{*}$ Correspondence:}

Lily Riggs, Rotman Research Institute,

Baycrest, 3560 Bathurst Street,

Toronto, ON, Canada M6A2E1.

e-mail:Iriggs@rotman-baycrest.on.ca
Research shows that memory for emotional aspects of an event may be enhanced at the cost of impaired memory for surrounding peripheral details. However, this has only been assessed directly via verbal reports which reveal the outcome of a long stream of processing but cannot shed light on how/when emotion may affect the retrieval process. In the present experiment, eye movement monitoring (EMM) was used as an indirect measure of memory as it can reveal aspects of online memory processing. For example, do emotions modulate the nature of memory representations or the speed with which such memories can be accessed? Participants viewed central negative and neutral scenes surrounded by three neutral objects and after a brief delay, memory was assessed indirectly via EMM and then directly via verbal reports. Consistent with the previous literature, emotion enhanced central and impaired peripheral memory as indexed by eye movement scanning and verbal reports. This suggests that eye movement scanning may contribute and/or is related to conscious access of memory. However, the central/peripheral tradeoff effect was not observed in an early measure of eye movement behavior, i.e., participants were faster to orient to a critical region of change in the periphery irrespective of whether it was previously studied in a negative or neutral context. These findings demonstrate emotion's differential influences on different aspects of retrieval. In particular, emotion appears to affect the detail within, and/or the evaluation of, stored memory representations, but it may not affect the initial access to those representations.

Keywords: emotion, memory tradeoff, eye movement monitoring

\section{INTRODUCTION}

For years, researchers have noted that emotionally arousing events are remembered better than neutral events (Cahill and McGaugh, 1998). However, emotion-enhanced memory does not always extend to all aspects of an event. Rather, emotion, or specifically negative emotion, may result in a central/peripheral tradeoff effect in memory: memory for central, emotional aspects of an event is enhanced, and memory for peripheral, non-emotional aspects of an event is impaired (for review, see Steblay, 1992; Levine and Edelstein, 2009). In other words, emotion affects the nature of one's memory representations for how a particular scene/event is remembered. While there are many studies showing the influence of emotion during encoding and consolidation (e.g., Cahill and McGaugh, 1998), it is unclear which aspects of retrieval are modulated by emotion. For example, in addition to the quality and/or the amount of details that are stored in memory, emotion may also affect the ease or speed at which such memories can be accessed and, further, whether such representations are subsequently available for conscious introspection.

Evidence in support of the central/peripheral tradeoff effect in memory has been derived exclusively from verbal reports (e.g., Loftus, 1979; Loftus et al., 1987; Christianson, 1992; Reisberg and Heuer, 2004; Kensinger et al., 2005, 2007), which provides a direct measure of the end product of a long stream of memory processing, but it cannot reveal processing differences online.
Previous studies show that online indices of memory, such as those garnered by eye movement monitoring (EMM), do not necessarily correspond to verbal reports memory (e.g., Ryan et al., 2000; Thornton and Fernandez-Duque, 2000, 2002; Ryan and Cohen, 2004; Laloyaux et al., 2006). Further, retrieval may occur in stages, therefore it may be useful to have a measure of memory that can evaluate retrieval throughout the process. The first stage of retrieval may reflect initial access to stored representations in memory. We have previously argued that access to memory representations occurs very early (within the first few fixations) and in an obligatory fashion, such that it is not affected by changes in task demands (e.g., Althoff and Cohen, 1999; Ryan et al., 2007a). Subsequent stages of retrieval may reflect a more evaluative process that depend critically on the quality of stored memory representation; this evaluative process allows for repetition and/or changes in the environment to be detected and may ultimately result in conscious access of the information (e.g., Ryan and Cohen, 2004; Hannula et al., 2007; Ryan et al., 2007a). On this view, emotion-impaired memory for peripheral information may be the result of difficulties in the initial access of memory, and/or differences in the amount of detail contained within those representations that are retrieved (e.g., Adolphs et al., 2001, 2005; Denburg et al., 2003). This would contribute to a more comprehensive understanding of how malleable the processes related to memory retrieval are, and how extensively emotion may influence 
memory, i.e., does it modulate seemingly "obligatory" processes during retrieval in the same manner as processes that are considered more evaluative?

In order to gain a more comprehensive understanding of the effect of emotion on memory, we employed measures derived from EMM as well as verbal reports to characterize retrieval processing differences as a function of emotion. In contrast to verbal reports, EMM can reveal aspects of mnemonic processing such as what aspects of a scene were subsequently remembered, and when this information was retrieved. Specifically, previous studies show that even when participants were not cued or instructed to make recognition memory judgments, the rate of overall sampling decreased for repeated versus novel scenes (repetition effect); and further, sampling increased for critical regions within a scene that had undergone a change in manipulated scenes compared to unchanged regions of repeated scenes (manipulation effect; e.g., Ryan et al., 2000; Ryan and Cohen, 2004). This shows that eye movement scanning behavior can be altered by prior experience, and by outlining where eye movements are attracted to within a scene that has undergone a change, it can reveal how detailed the memory representation is. Further, differences in eye movement behavior due to prior experience have been found to occur very early during processing (Althoff and Cohen, 1999; Ryan et al., 2007a,b) and in advance of explicit responding (Hannula et al., 2007), suggesting that EMM can reveal the time at which memories are initially accessed. Additionally, eye movement indices of memory may reveal that information has been retained in memory that is unavailable for conscious introspection (e.g., Althoff et al., 1998; Althoff and Cohen, 1999; Ryan et al., 2000; Thornton and Fernandez-Duque, 2000, 2002; Hollingworth et al., 2001; Hollingworth and Henderson, 2002; Ryan and Cohen, 2004; Laloyaux et al., 2006).

To address how emotion may affect the nature of, and access to memory representations for the central emotional and peripheral neutral information, we adapted an experimental paradigm which has been shown to elicit the central/peripheral tradeoff effect in memory when measured via verbal reports (Kensinger et al., 2007). During the study phase, participants studied a central picture that was either neutral or negatively arousing surrounded by three neutral everyday objects. After a brief delay, memory for central pictures and peripheral objects was assessed separately in the test phase in which previously viewed and novel central pictures, and previously viewed, manipulated, and novel peripheral objects were presented. Here, memory for the central pictures and peripheral objects was indexed by verbal reports and through changes in eye movement patterns as a function of prior exposure. Since the aim of the current study was to examine how emotion may affect what is retrieved from memory and when, we focus only on the results obtained during the retrieval phase of the experiment.

As shown in previous studies, evidence of a central/peripheral tradeoff in memory would be indexed by: (1) more accurate recognition, as measured via verbal reports, in identifying previously viewed negative versus neutral central pictures, and (2) conversely, more accurate recognition of peripheral objects that had been previously paired with neutral versus negative central pictures. Further, if emotion leads to retrieval advantages for the central negative versus neutral pictures, this would lead to a larger repetition effect (overall sampling decreases for previously viewed versus novel scenes) for central negative pictures. On the other hand, if emotion leads to retrieval disadvantages for the surrounding neutral information due to difficulties in access and/or less detailed memory representations, this would be manifested as: (1) faster orienting to a region of change among the peripheral objects previously paired with a neutral versus negative picture; and/or (2) increased viewing of manipulated versus repeated peripheral objects (manipulation effect) previously paired with neutral, but not negative pictures, respectively.

\section{MATERIALS AND METHODS PARTICIPANTS}

Twenty-four undergraduate students (mean age $=19.17$ years, three males; one left-handed) from the University of Toronto participated for course credit. All participants had normal neurological histories and normal or corrected-to-normal vision.

\section{STIMULI AND DESIGN}

The materials used to create the experimental displays consisted of 48 pictures taken from the International Affective Picture System (IAPS), of which 24 had a negative valence and 24 were of neutral valence (Lang et al., 1999), and 192 neutral objects (Hemera Photo Objects). The everyday objects were judged by the authors (Lily Riggs and Douglas A. McQuiggan) and two independent raters to be neutral and non-arousing. All pictures chosen from the IAPS set included people. The negative pictures had a more negative valence $(t=-17.03, p<0.001)$ and were more arousing $(t=14.02$, $p<0.0001)$ than the neutral pictures. Each display consisted of one picture in the center and three objects randomly placed in the periphery, which did not overlap in physical space or semantic meaning with the central element, but were always distinct and not relevant to the meaning of the central scene. A manipulated version was constructed for each display in which one of the three peripheral objects was replaced with a novel object. Each set of peripheral objects was counterbalanced across participants such that it was presented as paired with negative and neutral pictures equally. In the test block, the central pictures and peripheral objects were presented separately. Central pictures were either previously presented (repeated) or entirely new (novel). Peripheral objects contained the same three objects presented during the study phase (repeated), two previously studied objects and 1 novel object (manipulated) or three novel objects that were not presented during the study phase (novel). For all displays of peripheral objects in test block, a black box was placed in the location previously occupied by the central picture so that judgments of novelty/repetition could only be based on the peripheral objects rather than the central picture. Counterbalancing of the display occurred such that each version of the display appeared equally often in each experimental condition (repeated/novel for central pictures; repeated/manipulated/novel for peripheral objects) across participants.

\section{PROCEDURE}

Eye movements were measured throughout the study and test phases with a SR Research Ltd. Eyelink 1000 eye-tracking desktop monocular system and sampled at a rate of $1000 \mathrm{~Hz}$ with a spatial resolution $0.1^{\circ}$. A chin rest was used to limit head movements. A nine-point calibration was performed at the start of the 
experiment followed by a nine-point calibration accuracy test. Calibration was repeated if the average gaze error was greater than $1^{\circ}$ and if the error at any single point was more than $1.5^{\circ}$. Participants studied 32 randomly presented displays (16 negative, 16 neutral) once in each of two study blocks. The stimuli were repeated across two study blocks because previous EMM studies have shown that significant differences in viewing novel versus repeated stimuli manifested only after multiple exposures in which the trial duration was longer than in the current work (e.g., Althoff and Cohen, 1999; Ryan et al., 2000, 2007b). The displays were $1024 \times 768$ pixels in size and subtended approximately $33.4^{\circ}$ of visual angle when seated 25 inches from the monitor. Each display was presented for 2 s (e.g., Kensinger et al., 2005, 2007) followed by a 3-s inter-stimulus interval. Participants were instructed to freely view the scene. After a 10-min delay (approximately) in which participants completed a background information form, participants' memory for the peripheral objects and central pictures was assessed separately across four test blocks. Test blocks using indirect measures of memory were always assessed first, followed by test blocks that elicited direct verbal reports of memory. This was done in an effort to reduce the effect of verbal reports on eye movement responses (Yarbus, 1967; Ryan et al., 2000). To indirectly assess memory for the peripheral objects, participants were shown 16 previously studied, 16 manipulated, and 16 novel displays and were asked to again engage in free viewing while eye movements were monitored (Figure 1). Memory for the central pictures was assessed indirectly via EMM by presenting 32 previously studied and 16 novel pictures and asking participants subjects to engage in free viewing.

The same materials presented during the EMM test phase were repeated during the verbal response test phase. During the verbal response test blocks, participants were informed that they would be seeing the last two blocks of pictures again. In the first test block, participants had to indicate whether a set of peripheral objects was exactly the same as during the study sessions ("old"), had changed in some way ("manipulated") or was completely novel ("new"). In the second test block, participants had to indicate whether a central picture was the same (old) or different (new) from what they had seen during the study blocks.

\section{Analysis}

Eye movements were measured during the study and test phase. From the test phase, our analyses focused on the results from the repeated and manipulated peripheral objects as they were a direct test of emotional influences on memory (Kensinger et al., 2007). Analysis of eye movements was performed with respect to the experimenter-drawn interest areas corresponding to the location of central picture and peripheral objects. Eye movement measures of interest included the time of first fixation and the number of fixations into a region of interest. A fixation is defined as the absence of any saccade (e.g., the velocity of two successive eye movement samples exceeds $22^{\circ}$ s over a distance of $0.1^{\circ}$ ), or blink (e.g., pupil is

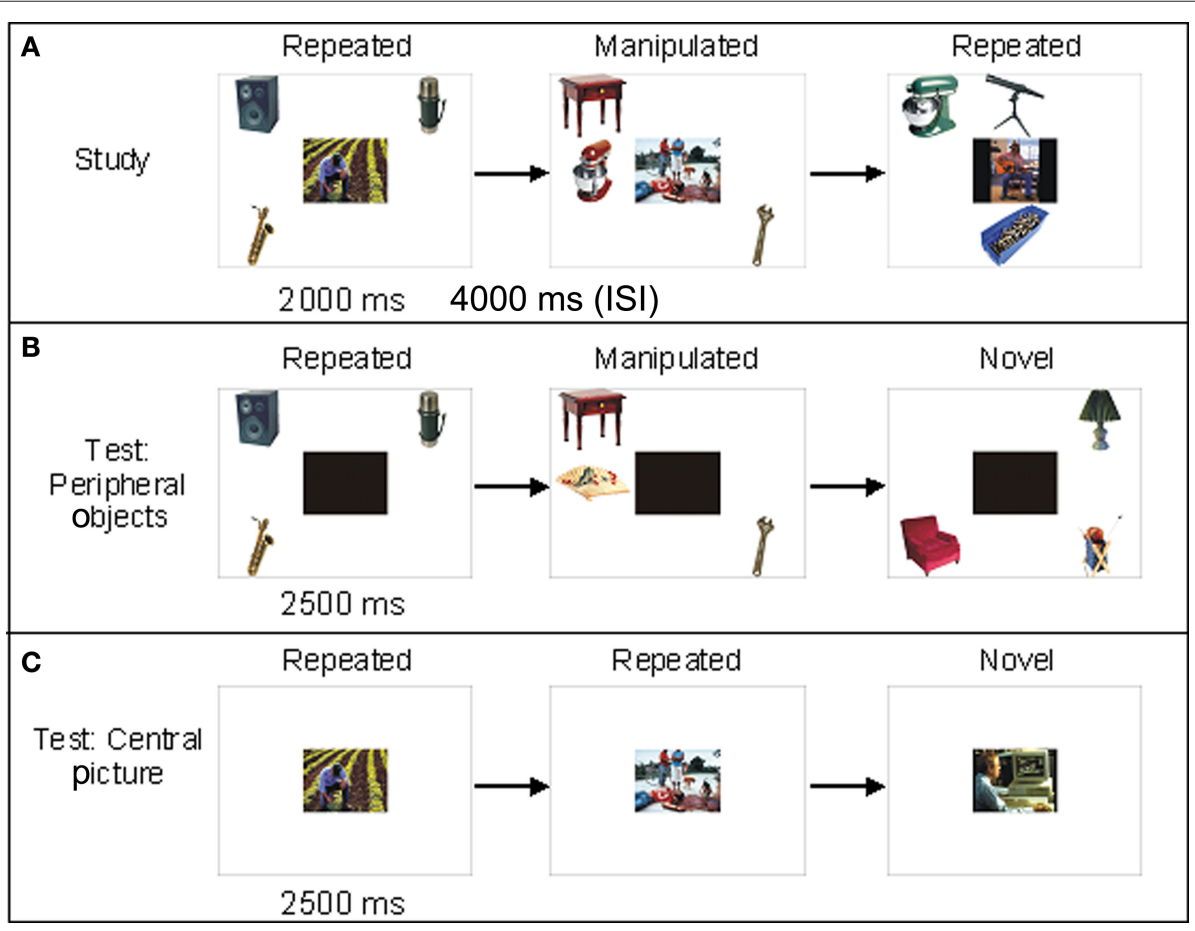

FIGURE 1 | Participants viewed negative and neutral central pictures paired with three everyday objects; each display was randomly presented once in each of two study blocks (A). During the test for peripheral objects, the central picture was blacked out so that only the peripheral objects were visible (B). Participants freely viewed repeated (three previously presented objects), manipulated (two previously presented, and one novel object) and novel (three never previously presented objects) peripheral objects. In the test for memory of central pictures, only the central picture was visible (C). This block consisted of repeated and novel pictures. 
missing for three or more samples) activity. The time of first fixation indicates how quickly overt attention was directed to a particular region of interest and provides an index of how quickly memory representations are accessed. The number of fixations indicates the amount of viewing directed within a particular region and provides a measure of the detail contained within the memory representation. Both EMM measures of time of first fixation and number of fixations provide an indirect measure of memory, as these measures can be collected without having participants simultaneously comment on the contents of their memories.

Evidence of memory during the test phase for the central pictures would be revealed as a decrease in the sampling of previously presented versus novel pictures (e.g., Althoff et al., 1998; Althoff and Cohen, 1999; Ryan et al., 2000). It is important to note that for central pictures, we examined eye movement differences between novel and repeated negative pictures and eye movement differences between novel and repeated neutral pictures. In other words, evidence of memory is manifested as changes in viewing between novel and previously viewed pictures, and not between negative and neutral pictures. Since participants always began each trial fixated in the center of the screen and the central region was the only filled region present on the screen, we examined only the number of fixations for central pictures. For the peripheral objects, a comparison of repeated versus novel/manipulated peripheral objects provided evidence for the time at which memory representations may be accessed and the quality of those stored representations (e.g., Ryan and Cohen, 2004). This was examined as a proportion of difference in viewing the critical object in repeated and manipulated arrays with reference to viewing of the critical object in novel arrays as a baseline. The critical object in novel object arrays was never associated with a neutral or negative central picture and served as a baseline to correct for individual differences in viewing. Evidence of impaired access to peripheral objects as a result of emotion would be manifested by slower orienting to the critical object (the novel object among two repeated objects) in manipulated displays versus the exact same "critical" object in repeated displays which had not undergone a change for peripheral object that had been paired with negative versus neutral pictures. Evidence of a less detailed memory representation as a result of emotion would be manifested by a lack of difference in the number of fixations directed to the critical object in manipulated versus repeated displays for those peripheral objects that had been paired with a negative, but not neutral, picture. In order to control for stimulus specific effects, the critical object appeared as a novel object within a manipulated display, as a repeated object within a repeated display, and as a novel object within a novel display across participants. Additionally, the presentation of central pictures as novel or previously viewed was counterbalanced across participants, thus any differences in viewing was the result of the participants' prior viewing history (Ryan et al., 2000, 2007b).

Recognition accuracy was measured as the proportion of correct responses to novel and repeated central pictures, and novel, repeated and manipulated peripheral objects. Reported hits for central pictures were corrected for false alarms. Reported hits to repeated and manipulated peripheral objects are presented uncorrected for false alarm rates as novel peripheral objects were not presented with emotional/neutral images.

\section{RESULTS \\ CENTRAL PICTURES}

\section{Eye movement measures}

Eye movements were analyzed with respect to the interest area corresponding to the location of the central picture. The raw means and standard errors for the number of fixations made to the central pictures are presented in Table 1. Differences in the number of fixations directed to novel versus repeated pictures were calculated using novel pictures as the baseline. We then used paired-sample $t$-tests to determine whether this difference in viewing was significantly different from 0 and modulated by emotion (negative versus neutral). Consistent with the notion that emotion enhances memory, differences in viewing novel versus repeated pictures was significantly different from 0 when the pictures were negative $(t(23)=3.01, p<0.01)$, but not when they were neutral $(t(23)=1.38, p=0.18)$. Specifically, participants directed fewer fixations to repeated versus novel pictures only when they were negative. A direct comparison of viewing of negative and neutral central pictures was not significant $(t(23)=0.37, p=0.72)$.

Table 1 | Means and standard errors for eye movement measures for viewing of the critical object in the periphery and central scenes during test session.

Critical peripheral object

\begin{tabular}{|c|c|c|c|c|c|c|}
\hline Measures & \multicolumn{3}{|c|}{ Neutral } & \multicolumn{3}{|c|}{ Negative } \\
\hline Number of fixations (\#): & $2.06(0.09)$ & $2.25(0.14)$ & $1.88(0.10)$ & $\mathrm{N} / \mathrm{A}$ & $2.10(0.13)$ & $1.99(0.11)$ \\
\hline
\end{tabular}

Central pictures

\begin{tabular}{|c|c|c|c|c|}
\hline & \multicolumn{2}{|c|}{ Neutral } & \multicolumn{2}{|c|}{ Negative } \\
\hline & Novel & Repeated & Novel & Repeated \\
\hline
\end{tabular}




\section{Verbal recognition reports}

Verbal recognition for the central pictures was analyzed using a paired-sample $t$-test examining accuracy for repeated negative and neutral pictures. When hit rates were corrected by false alarms, participants were more accurate in identifying repeated pictures when they were negative versus when they were neutral $(t(23)=2.86, p<0.01)$. All relevant means and standard errors are presented in Table 2.

In summary, when memory was measured indirectly via EMM at the test phase, eye movement patterns distinguished between repeated and novel pictures when they were negative, but not when they were neutral pictures. Consistent with this, emotion was also found to enhance recognition memory for repeated central pictures when measured directly via verbal reports.

\section{PERIPHERAL OBJECTS}

\section{Eye movement measures}

Eye movements were analyzed with respect to the interest area corresponding to the location of the critical object which was the novel object among two repeated objects in the manipulated arrays and the corresponding object in the repeated and novel object arrays. Proportion of difference in viewing of the critical object between manipulated and repeated displays relative to novel displays reveals the extent to which information regarding the peripheral objects was retained in memory (Ryan et al., 2000; Ryan and Cohen, 2004).

Eye movement measures to the critical object were analyzed using separate $2 \times 2$ repeated measures ANOVA using emotion (negative, neutral) and object array type (manipulated, repeated) as withinsubject factors. All relevant raw means and standard errors are presented in Table 1. Consistent with the notion that EMM measures are sensitive to prior experience, there was a significant main effect of object array type (both measures: $F(1,23)=7.47, p=0.01, d=0.25$ ). Participants were faster to fixate, and directed more viewing to the critical object when it appeared in a manipulated versus a repeated display, regardless of whether that set of peripheral objects had been previously paired with a negative or neutral central picture. The main effect of emotion on eye movement behavior was not significant (time of first fixation: $F(1,23)=0.16, p=0.69, d=0.01$; number of fixations: $F(1,23)=0.11, p=0.75, d=0.004)$. There was a significant interaction for the number of fixations $(F(1,23)=4.11, p=0.05, d=0.15$; Figure 2); participants directed more fixations to the critical object when it appeared in a manipulated compared to a repeated array if the objects had been previously paired with a neutral central picture $(t(23)=2.94, p=0.007)$, but not when it had been paired with a negative central picture $(t(23)=1.23, p=0.23)$. The interaction between emotion and type was not significant for the time of first fixation $(F(1,23)=0.58, p=0.46, d=0.02)$, suggesting that while emotion affected the overall quality (i.e., number of fixations) of memory for peripheral objects, it did not affect access to, and early indicators of, memory for detecting which peripheral object had been altered, which occurred approximately $800 \mathrm{~ms}$ following stimulus onset. ${ }^{1}$

\section{Verbal recognition reports}

Verbal recognition accuracy was analyzed with repeated measures ANOVA using emotion (negative, neutral) and peripheral object array type (manipulated, repeated) as within-subject factors. For accuracy, the main effect of object type was significant $(F(1,23)=61.14$, $p<0.0001, d=0.73)$. In other words, participants were more accurate in identifying repeated versus manipulated object arrays. The main effect of emotion was marginally significant $(F(1,23)=3.59, p=0.07$, $d=0.14$ ); participants were more accurate in classifying peripheral objects as either repeated or manipulated if they had been previously paired with a neutral central picture rather than a negative central picture. Planned contrasts revealed that participants were significantly more accurate in identifying manipulated peripheral objects if they were previously paired with a neutral central picture versus a negative central picture $(t(23)=2.19, p<0.05)$. Emotion did not modulate accuracy for repeated peripheral objects $(t(23)=0.30, p=0.77)$. All relevant means and standard errors are presented in Table 2.

In summary, indirect measures of memory as indexed by EMM revealed that early eye movement patterns distinguished between manipulated and repeated object arrays irrespective of whether they were previously paired with a negative or neutral picture. In contrast, viewing of the periphery was modulated by emotion and only distinguished between manipulated and repeated object arrays of those previously paired with a neutral picture. Consistent with this, emotion was also found to impair recognition memory, as indexed by verbal reports, for detecting a change in the periphery.

${ }^{1}$ It should be noted that the same pattern of results was found when we examined the raw values resulting from the EMM measures.

Table 2 | Mean responses and standard errors for peripheral objects and central pictures.

Peripheral objects

\begin{tabular}{|c|c|c|c|c|c|c|}
\hline & \multicolumn{3}{|c|}{ Neutral } & \multicolumn{3}{|c|}{ Negative } \\
\hline & Novel & Manipulated & Repeated & Novel & Manipulated & Repeated \\
\hline
\end{tabular}

Central pictures

\begin{tabular}{|c|c|c|c|c|c|c|}
\hline & \multicolumn{3}{|c|}{ Neutral } & \multicolumn{3}{|c|}{ Negative } \\
\hline & Novel & Repeated & Repeated (Corrected) & Novel & Repeated & Repeated (Corrected) \\
\hline
\end{tabular}




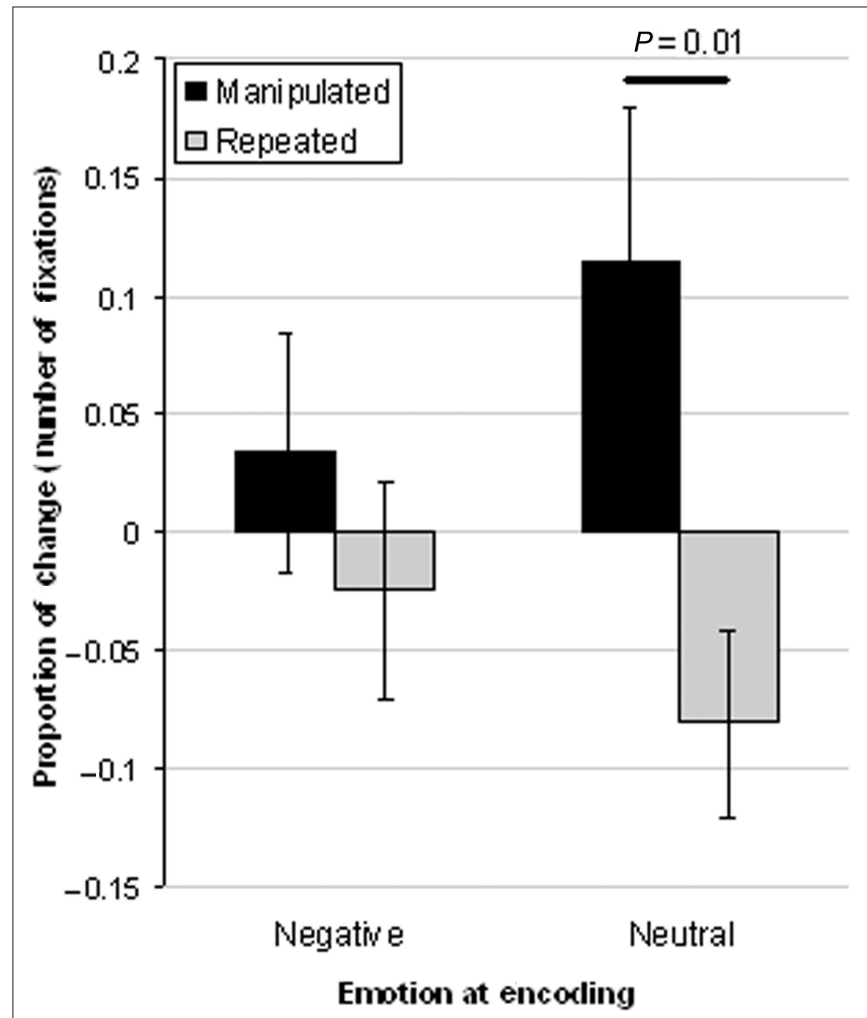

FIGURE 2 |The proportion of change in viewing the critical object in a manipulated and repeated object array relative to a novel object array. Participants directed more fixations to the critical object in manipulated versus repeated object arrays for objects previously encoded in a neutral, but not in a negative context.

\section{RELATION BETWEEN VERBAL REPORTS AND EYE MOVEMENT DATA}

In further support of the finding that there may be a dissociation between memory measured by verbal reports versus EMM, we also examined EMM for half of the participants who showed the strongest emotion-modulated effect in verbal memory, i.e., those who showed the largest difference in correctly identifying peripheral objects previously paired with negative $(M=0.11, \mathrm{SEM}=0.03)$ versus neutral pictures $(M=0.34, \mathrm{SEM}=0.03 ; t(11)=5.14, p<0.0001)$. Despite this strong tradeoff effect in memory as measured by verbal reports, the same tradeoff effect was not observed in EMM measures. Specifically, a repeated measures ANOVA using emotion (negative, neutral) and object type (manipulated, repeated) did not reveal significant main effects of emotion for either of the EMM measures (time of first fixation: $F(1,11)=0.43, p>0.1, d=0.04$; number of fixations: $F(1,11)=1.43, p>0.1, d=0.12)$ nor did it reveal a significant interaction between emotion and object type (time of first fixation: $F(1,11)=0.66, p>0.1, d=0.06$; number of fixations: $F(1,11)=1.67, p>0.1, d=0.13)$. However, consistent with previous results, there was a significant main effect of object type for time of first fixation $(F(1,11)=6.35, p<0.05, d=0.37)$ such that participants were quicker to fixate on manipulated versus repeated objects regardless of whether the objects were previously paired with neutral or negative central pictures. The main effect of type was not significant for the number of fixations $(F(1,11)=2.87$, $p>0.1, d=0.21)$. This suggests that the strongest dissociation between verbal reports and EMM may be observed in measures of early viewing such as the time of first fixation, which is modulated by prior experience, but not emotion. ${ }^{2}$

\section{DISCUSSION}

The presence of emotional stimuli results in a central/peripheral tradeoff effect in memory (e.g., Wessel and Merckelbach, 1997; Kensinger et al., 2005, 2007). Prior work suggests that emotions change the nature of memory representations for the emotioneliciting stimulus and surrounding neutral information. However, this has only been explored using explicit verbal reports which reveal the end product of what is held in memory and cannot speak to how quickly one may be able to access stored representations. Using measures derived from EMM and verbal reports, the present work examined whether the presence of emotional stimuli led to differences in the speed at which memory representations could subsequently be accessed at retrieval, and whether there were differences in the details maintained within those representations. To the best of our knowledge, the present study is the first to examine these issues regarding the influence of emotion on distinct aspects of retrieval. In the next section, we discuss our results in light of prior findings regarding the central peripheral tradeoff in memory, and how the current work may inform theories regarding the influence of emotion on memory.

The use of EMM allows for the examination of how early viewing may be modulated by prior experience and whether this was influenced by the emotional context in which the information was originally encoded. Consistent with previous research, the current results showed that an early indicator of memory (i.e., time of first fixation to an altered region) was modulated by prior experience (e.g., Althoff and Cohen, 1999; Henderson et al., 2003; Ryan and Cohen, 2004). Specifically, participants were approximately $105 \mathrm{~ms}$ faster to fixate on the peripheral critical object when it was manipulated compared to when it was repeated which suggests that participants were able to encode, store, and at least to some degree, access information about these peripheral objects during the test phase such that early eye movement behavior was altered (Parker, 1978). The difference in the time of first fixation occurred as early as $800 \mathrm{~ms}$, which is rapid considering that participants did not know which object arrays would be manipulated and where the critical object would appear. Critically, this early indicator of memory differentiated between manipulated and repeated object arrays irrespective of the emotional context in which the objects were originally encoded. Thus, contrary to the notion that emotion impairs memory for information in the periphery, the current results show that emotion did not modulate early access of memory when measured indirectly via EMM.

In addition to examining an early indicator of memory via EMM, the current study also examined viewing patterns during the entire presentation period, i.e., number of fixations. Consistent with the central/peripheral tradeoff effect in memory, viewing patterns showed that emotion-enhanced memory for central pictures and

${ }^{2}$ Since accuracy for central pictures was at ceiling, there was not enough variability to conduct the same type of analysis to examine the relation between verbal reports and eye movement behavior for central pictures. 
impaired memory for peripheral objects. Specifically, it was found that viewing of central pictures was characterized by a repetition effect, i.e., a decrease in the number of fixations in viewing repeated versus novel scenes (e.g., Althoff et al., 1998; Althoff and Cohen, 1999; Ryan et al., 2000, 2007b) for negative, but not neutral central pictures. Previous studies have shown that significant differences in viewing novel versus repeated stimuli largely occur only after multiple exposures in which the trial duration was longer than in the current work (e.g., Althoff and Cohen, 1999; Ryan et al., 2000, 2007b). Thus, it is likely that the EMM metric did not distinguish between novel and repeated neutral central pictures because more repetitions were required before such differences in eye movement behavior could manifest. Despite this, eye movement behavior did distinguish between novel and repeated negative pictures, which suggests that emotion does not only enhance the probability that the picture will later be remembered, it also suggests that emotion may enhance the speed at which a lasting memory representation is formed. It is important to note that the repetition effect found in the eye movement behavior for viewing negative central pictures may represent the contributions of perceptual fluency rather than (or in addition to) declarative/relational memory. For example, repetition effects have been demonstrated in amnesic patients who have compromised medial temporal lobe systems (e.g., Althoff et al., 1998; Ryan et al., 2000), and intact repetition effects have been observed in healthy older adults in whom compromised medial temporal lobe function has been implicated (Driscoll et al., 2003; Ryan et al., 2007b).

For peripheral objects, participants directed significantly more fixations to the critical object of manipulated versus repeated displays (manipulation effect) if the objects were previously studied with a neutral central picture, but not when the peripheral objects were studied with a negative central picture. The finding that more fixations were directed to the manipulated versus repeated critical object is consistent with previous eye movement studies that have reported an increase in viewing for regions of change (e.g., Ryan et al., 2000, 2007b; Ryan and Cohen, 2004). Such effects have been reported irrespective of task demands and have been found to precede behavioral responding, which suggest that such eye movement behaviors may ultimately culminate in the conscious access of previously learned information. It is possible that it is only through an increase in the amount of viewing to, and investigation of, a region of change that allows one to not only notice a change, but also be able to explicitly identify what had been changed and how. Further, on this view, it is likely that a manipulated versus repeated scene may require a more extensive comparison process between the presented external stimulus and the internal memory representation, leading to an increase in viewing (see Ryan and Cohen, 2004, for further discussion). In addition, such an increase in viewing may also represent the re-binding and/or the updating of memory representations. Thus, although early access to memory was not modulated by emotion, the quality and/or the amount of details contained within the memory, as indexed by the amount of sampling, was modulated by the emotional history of the retrieved information. This suggests that although emotion may lead to a more impoverished memory representation for information in the periphery, it may not impair one's ability to access that information during the retrieval phase, however poor in quality those representations may be. In contrast to the repetition effect found for viewing of novel and repeated central pictures, the manipulation effect found for viewing of manipulated versus repeated peripheral objects likely reflect the influence of emotion on the representations that are declarative/relational in nature; as eye movement indices of detection of a manipulation are impaired in amnesic patients (Ryan et al., 2000) and older adults who presumably have a compromised medial temporal lobe system (Ryan et al., 2007b). Altogether, it would appear that emotion affects the formation of (detail contained within) multiple memory representations, including those that would contribute to perceptual fluency and those that are declarative/relational in nature and which support identification of a change by the eyes. However, it does not appear that emotion affects the speed with which such representations are accessed.

Consistent with the notion that measures of sampling of the critical region may contribute and/or is related to the final output of memory processing, direct measures of memory obtained through verbal reports showed that emotion-enhanced memory for central pictures and impaired memory for peripheral objects. Specifically, participants were more accurate to identify repeated negative versus neutral central pictures, and less accurate to detect a change in the periphery if the peripheral objects were previously studied with a negative compared to a neutral picture (e.g., Wessel and Merckelbach, 1997; Brown, 2003; Kensinger et al., 2005). Interestingly, while emotion-impaired participants' ability to detect a change in the peripheral objects, it did not modulate their ability to identify repeated peripheral objects (see: Kensinger et al., 2005, 2007). A possible reason for this is that whereas previous studies have presented the stimuli once during the encoding phase, the present study presented the stimuli twice across two study blocks. It is possible that by repeating the stimuli, the central/peripheral tradeoff effect in memory was not as robust as it would have been had the stimuli only been presented once. There is some indication in the literature that the central/peripheral tradeoff effect in memory is sensitive to methodological parameters such as the duration of exposure to the stimuli, specificity of the information interrogated during the test phase and the length of time between encoding and retrieval (e.g., Burke et al., 1992; Christianson, 1992; Steblay, 1992). However, despite having presented the stimuli twice during the study blocks, we still observed an influence of emotion on the memory for the peripheral objects. Detection of a manipulation within the peripheral objects may require a more detailed declarative/relational memory representation as participants need to be able to identify a critical novel object among two previously viewed objects. Thus, the current results may suggest that memory for specific details in the periphery is more sensitive to emotional modulation than memory for the gist of information (Adolphs et al., 2001, 2005; Denburg et al., 2003).

The results of this study showed that while emotion (here, negative emotion) did not modulate early indicators of, or access to memory, it led to a central/peripheral tradeoff in memory as indexed by sampling of the stimulus and by verbal reports. These differences in the influence of emotion may be due to differences in what such changes in eye movement measures and verbal reports represent; specifically, the early online use of memory versus the quality of stored memory representations, and subsequent conscious access 
to those representations. An important question that remains is how emotion may influence the quality and/or amount of details stored in memory. It is often argued such differences in memory are the result of emotion-modulated differences during the encoding phase. However, there has not been a complete examination of whether such differences in attention during the encoding phase are related to subsequent memory (see Christianson et al., 1991; Wessel et al., 2000). In a recent paper by Riggs and colleagues (in press), the researchers used EMM as an index of overt attention allocation, and mediation analysis to determine whether differences in attention were related to subsequent memory. It was found that contrary to previous assumptions, differences in attention during the encoding phase did not fully explain the central/peripheral tradeoff effect in verbal reports memory. These findings suggest that the differential influence of negative emotion on central versus peripheral memory may result from other cognitive influences in addition to visual attention, or on post-encoding processes. Alternatively, it could also be argued that while EMM provides a reliable measure of overt attention, it cannot capture processes related to covert attention which can be decoupled from overt attention (e.g., Posner, 1980; Rowe et al., 2007). Future studies can more systematically differentiate between these two contributing factors.

\section{REFERENCES}

Adolphs, R., Denburg, N. L., and Tranel, D. (2001). The amygdala's role in longterm declarative memory for gist and detail. Behav. Neurosci. 115, 983-992.

Adolphs, R., Tranel, D., and Buchanan, T. W. (2005). Amygdala damage impairs emotional memory for gist but not details of complex stimuli. Nat. Neurosci. 8, 512-518.

Althoff, R., Cohen, N. J., McConkie, G., Wasserman, S., Maciukenas, M., Azen, R., and Romine, L. (1998). "Eye movement-based memory assessment," in Current Oculomotor Research: Physiological and Psychological Aspects, eds W. Becker, H. Deubel, and T. Mergner (New York: Plenum Publishers), 293-302.

Althoff, R. R., and Cohen, N. J. (1999). Eye-movement-based memory effect: a reprocessing effect in face perception. J. Exp. Psychol. Learn. Mem. Cogn. 25, 997-1010.

Brown, J. M. (2003). Eyewitness memory for arousing events: putting things in context. Appl. Cogn. Psychol. 17, 93-106.

Burke, A., Heuer, F., and Reisberg, D. (1992). Remembering emotional events. Mem. Cognit. 20, 277-290.

Cahill, L., and McGaugh, J. L. (1998). Mechanisms of emotional arousal and lasting declarative memory. Trends Neurosci. 21, 294-299.

Christianson, S.-A. (1992). Emotional stress and eyewitness memory: a critical review. Psychol. Bull. 112, 284-309.
Christianson, S.-A., Loftus, E. F., Hoffman, H., and Loftus, G. R. (1991). Eye fixations and memory for emotional events. J. Exp. Psychol. Learn. Mem. Cogn. 17, 693-701.

Denburg, N. L., Buchanan, T. W., Tranel, D., and Adolphs, R. (2003). Evidence for preserved emotional memory in normal older persons. Emotion 3, 239-253.

Driscoll, I., Hamilton, D. A., Petropoulos, H., Yeo, R. A., Brooks, W. M., Baumgartner, R. N., and Sutherland, R. J. (2003). The aging hippocampus: cognitive, biochemical and structural findings. Cereb. Cortex 13, 1344-1351.

Hannula, D. E., Ryan, J. D., Tranel, D., and Cohen, N. J. (2007). Rapid onset relational memory effects are evident in eye movement behavior, but not in hippocampal amnesia. J. Cogn. Neurosci. 19, 1690-1705.

Henderson, J. M., Williams, C. C., Castelhano, M. S., and Falk, R. J. (2003). Eye movements and picture processing during recognition. Percept. Psychophys. 65, 725-734.

Hollingworth, A., and Henderson, J. M. (2002). Accurate visual memory for previously attended objects in natural scenes. J. Exp. Psychol. Hum. Percept. Perform. 28, 113-136.

Hollingworth, A., Williams, C. C., and Henderson, J. M. (2001). To see and remember: Visually specific information is retained in memory from previously attended objects in natural scenes. Psychon. Bull. Rev. 8, 761-768.

In summary, the current findings suggest that emotion does not modulate all aspects of retrieval. Access to previously formed memory representations occurred early, without regard to the nature of the information that is contained therein. Together, with our previous work that suggests initial access to memory occurs despite differences in task demands (e.g., Ryan et al., 2007a), and even when such information is not relevant for the task at hand (Ryan et al., 2007a), we propose that retrieval of previously stored memory representations occurs in an obligatory fashion, despite the valence of the stored information. By contrast, emotion impacts the detail and/or amount of information that is maintained in memory and the likelihood that there will be conscious access to that information. Thus, the more evaluative components of memory (formation and) retrieval are impacted by emotional valence.

\section{ACKNOWLEDGMENTS}

This work was supported by funding from the Natural Sciences and Engineering Research Council of Canada (Jennifer D. Ryan, Adam K. Anderson), the Canada Research Chairs Program (Jennifer D. Ryan), the Canadian Foundation for Innovation (Jennifer D. Ryan), and a Postgraduate Scholarship from the Natural Sciences and Engineering Research Council of Canada (Lily Riggs).

Kensinger, E. A., Gutchess, A. H., and Schacter, D. L. (2007). Effects of aging and encoding instructions on emotion-induced memory trade-offs. Psychol. Aging 22, 781-795.

Kensinger, E. A., Piguet, O., Krendl, A C., and Corkin, S. (2005). Memory for contextual details: effects of emotion and aging. Psychol. Aging 20, 241-250.

Laloyaux, C., Devue, C., Doyen, S. David, E., and Cleeremans, A. (2006). Undetected changes in visible stimuli influence subsequent decisions. Conscious. Cogn. 17, 646-656.

Lang, P. J., Bradley, M. M., and Cuthbert, B. N. (1999). International Affective Picture System (IAPS): Instruction Manual and Affective Ratings. Tech. Rep. No. A-4 Gainesville: University of Florida.

Levine, L. J., and Edelstein, R. S. (2009) Emotion and memory narrowing: a review and goal- relevance approach. Cogn. Emot. 23, 833-875.

Loftus, E. (1979). Eyewitness reliability. Science 205, 386-387.

Loftus, E. A., Loftus, G. R., and Messo, J. (1987). Some facts about "weapon focus". Law Hum. Behav. 11, 55-62.

Parker, R. E. (1978). Picture processing during recognition. J. Exp. Psychol. Gen. 4, 284-292.

Posner, M. I. (1980). Orienting of attention. Q. J. Exp. Psychol. 32, 3-25.

Reisberg, D., and Heuer, F. (2004). "Remembering emotional events," in Memory and Emotion, eds D. Reisberg and P. Hertel (New York: Oxford University Press), 3-41.
Riggs, L., McQuiggan, D. M., Farb, N., Anderson,A.K., and Ryan,J.D. (in press). The role of overt attention in emotion modulated memory. Emotion.

Rowe, G., Hirsh, J. B., and Anderson, A. K. (2007). Positive affect increases the breadth of attentional selection. Proc Natl. Acad. Sci. U. S. A. 104, 383-388.

Ryan, J. D., Althoff, R. R., Whitlow, S., and Cohen, N. J. (2000). Amnesia is a deficit in declarative (relational) memory. Psychol. Sci. 11, 454-461.

Ryan, J. D., and Cohen, N. J. (2004). The nature of change detection and online representation of scenes. $J$. Exp. Psychol. Hum. Percept. Perform. 30, 988-1015.

Ryan, J. D., Hannula, D. E., and Cohen, N. J. (2007a). The obligatory effects of memory on eye movements. Memory $15,508-525$.

Ryan, J.D., Leung, G., Turk-Browne, N, B., and Hasher, L. (2007b). Assessment of age-related changes in inhibition and binding using eye movement monitoring. Psychol. Aging 22, 239-250.

Steblay, N. M. (1992). A meta-analytic review of the weapon focus effect. Law Hum. Behav. 16, 413-424.

Thornton, I.M., and Fernandez-Duque, D. (2000). An implicit measure of undetected change. Spat. Vis. 14, 21-44.

Thornton, I. M., and Fernandez-Duque, D. (2002). Converging evidence for the detection of change without awareness. Prog. Brain Res. 140, 99-118.

Wessel, I., and Merckelbach, H. (1997). The impact of anxiety on memory for 
details in spider phobics. Appl. Cogn. Psychol. 11, 223-231.

Wessel, I., van der Kooy, P., and Merckelbach, H. (2000). Differential recall of central and peripheral details of emotional slides is not a stable phenomenon. Memory 8, 95-100.

Yarbus, A. L. (1967). Eye Movements and Vision. New York: Plenum Press.
Conflict of Interest Statement: The authors declare that the research was conducted in the absence of any commercial or financial relationships that could be construed as a potential conflict of interest.

Received: 23 April 2010; accepted: 01 November 2010; published online: 25 November 2010.
Citation: Riggs L, Mcquiggan DA, Anderson AK and Ryan JD (2010) Eye movement monitoring reveals differential influences of emotion on memory. Front. Psychology 1:205. doi: 10.3389/ fpsyg.2010.00205

This article was submitted to Frontiers in Emotion Science, a specialty of Frontiers in Psychology.
Copyright (c) 2010 Riggs, Mcquiggan, Anderson and Ryan. This is an openaccess article subject to an exclusive license agreement between the authors and the Frontiers Research Foundation, which permits unrestricted use, distribution, and reproduction in any medium, provided the original authors and source are credited. 